\title{
Oxytocin Enhances Amygdala-Dependent, Socially Reinforced Learning and Emotional Empathy in Humans
}

\author{
René Hurlemann, ${ }^{1 \star}$ Alexandra Patin, ${ }^{1 \star}$ Oezguer A. Onur, ${ }^{1}$ Michael X. Cohen, ${ }^{2}$ Tobias Baumgartner, ${ }^{1}$ Sarah Metzler, ${ }^{1}$ \\ Isabel Dziobek, ${ }^{3}$ Juergen Gallinat, ${ }^{4}$ Michael Wagner, ${ }^{1}$ Wolfgang Maier, ${ }^{1}$ and Keith M. Kendrick ${ }^{5}$ \\ ${ }^{1}$ Department of Psychiatry, University of Bonn, 53105 Bonn, Germany, ${ }^{2}$ Department of Psychology, University of Arizona, Tucson, Arizona 85721, \\ ${ }^{3}$ Department of Educational Science and Psychology, University of Berlin, 14195 Berlin, Germany, ${ }^{4}$ Department of Psychiatry and Psychotherapy, Charité \\ University Medicine Berlin, 10117 Berlin, Germany, and ${ }^{5}$ Cognitive and Systems Neuroscience, The Babraham Institute, Babraham, Cambridge CB22 3AT, \\ United Kingdom
}

Oxytocin (OT) is becoming increasingly established as a prosocial neuropeptide in humans with therapeutic potential in treatment of social, cognitive, and mood disorders. However, the potential of OT as a general facilitator of human learning and empathy is unclear. The current double-blind experiments on healthy adult male volunteers investigated first whether treatment with intranasal 0T enhanced learning performance on a feedback-guided item-category association task where either social (smiling and angry faces) or nonsocial (green and red lights) reinforcers were used, and second whether it increased either cognitive or emotional empathy measured by the Multifaceted Empathy Test. Further experiments investigated whether OT-sensitive behavioral components required a normal functional amygdala. Results in control groups showed that learning performance was improved when social rather than nonsocial reinforcement was used. Intranasal OT potentiated this social reinforcement advantage and greatly increased emotional, but not cognitive, empathy in response to both positive and negative valence stimuli. Interestingly, after OT treatment, emotional empathy responses in men were raised to levels similar to those found in untreated women. Two patients with selective bilateral damage to the amygdala (monozygotic twins with congenital Urbach-Wiethe disease) were impaired on both OT-sensitive aspects of these learning and empathy tasks, but performed normally on nonsocially reinforced learning and cognitive empathy. Overall these findings provide the first demonstration that OT can facilitate amygdala-dependent, socially reinforced learning and emotional empathy in men.

\section{Introduction}

There is unprecedented interest in the role of the neuropeptide oxytocin (OT) as a key effector of human social behaviors (Lee et al., 2009). Substantial evidence implicates OT in facilitating human bonding and trust (Kosfeld et al., 2005; Baumgartner et al., 2008), but its potential as a cognitive enhancer or empathogen is still unclear. With learning and memory, OT has been claimed to have both pro-mnestic (Guastella et al., 2008b; Savaskan et al., 2008; Rimmele et al., 2009) and amnestic (Ferrier et al., 1980; Fehm-Wolfsdorf et al., 1984; Bruins et al., 1992; Heinrichs et al., 2004) effects. One hypothesis reconciling these contradictory

\footnotetext{
Received Nov. 9, 2009; revised Jan. 26, 2010; accepted Feb. 17, 2010.

R.H. was supported by a German Research Foundation Grant (HU1302/2-2) and by a Starting Independent Researcher Grant jointly provided by the Ministry of Innovation, Science, Research, and Technology of the State of North Rhine-Westphalia and the University of Bonn. K.M.K. was supported by the Biotechnology and Biological Sciences Research Council. *R.H. and A.P. contributed equally to this article. A.P.'s contribution was used toward her thesis for the International Master in Affective Neuroscience. We thank D. Talmi for superb methodological advice, I. Frommann and D. Patin for excellent technical assistance, and A. Edele and C. Montag for generously providing the Multifaceted Empathy Test.

The authors declare no competing financial interests.

This article is freely available online through the J Neurosci Open Choice option.

Correspondence should be addressed to either of the following: René Hurlemann, Department of Psychiatry, University of Bonn, 53105 Bonn, Germany, E-mail: renehurlemann@me.com; or Keith M. Kendrick, Cognitive and Systems Neuroscience, The Babraham Institute, Babraham, Cambridge CB22 3AT, UK, E-mail: keith.kendrick@ bbsrc.ac.uk.

D0I:10.1523/JNEUROSC1.5538-09.2010

Copyright $\odot 2010$ the authors $\quad 0270-6474 / 10 / 304999-09 \$ 15.00 / 0$
}

findings is that OT may specifically promote learning that is socially reinforced. Pioneering psychological studies suggest that social reinforcement is a potent facilitator of human learning (Allport, 1920; Gates and Rissland, 1923; Dashiell, 1930; Zajonc, 1965). We therefore investigated whether OT selectively improved learning when social as opposed to nonsocial feedback is used in a reinforcement associative learning task (RALT).

In animals, the empathogenic agent 3,4-methylenedioxymethamphetamine (MDMA, ecstasy) releases OT, and an OT antagonist prevents its prosocial effects (Thompson et al., 2007). In humans, OT enhances inference of emotional states (Domes et al., 2007a) as well as trust in (Kosfeld et al., 2005; Baumgartner et al., 2008) and generosity toward (Zak et al., 2007) others, suggesting that OT has empathogenic properties and could influence behavior in the context of social bonding. Related to this, studies have shown that OT reduces both social anxiety (Heinrichs et al., 2003) and amygdala responses to fearful faces (Kirsch et al., 2005), implying a possible trade-off of OT and amygdala responsivity and in turn a greater willingness to trust, and consequently bond to, other people. Even though empathy is influenced by OT receptor (OTR) genetic variation (Rodrigues et al., 2009) and closely related to prosocial and bonding behaviors that have been suggested to be enhanced by OT (Zak et al., 2007), there is as yet no direct experimental evidence for a modulation of empathy by OT (Singer et al., 2008). One prevalent concept distinguishes cognitive (recognizing emotional states in others) from emo- 
tional (sharing experiences of emotional states perceived in others) empathy (Rogers et al., 2007) (see also Adolphs, 2009). Consequently, we used an extended version of the "Multifaceted Empathy Test” (MET) providing behavioral indices of both cognitive and emotional empathy (Dziobek et al., 2008) to explore potential effects of OT.

While the OTR is widely distributed in brain regions implicated in cognition and emotion (Yoshimura et al., 1993; Hein and Singer, 2008), we have focused on the amygdala, building on previous findings showing substantial OTR expression in the rat amygdala (Insel and Shapiro, 1992; Veinante and Freund-Mercier, 1997) and where OT regulates neuronal activity (Huber et al., 2005). The social recognition memory-enhancing effects in humans (Guastella et al., 2008b; Savaskan et al., 2008; Rimmele et al., 2009) and other animals (Ferguson et al., 2001) as well as the anxiolytic effects of OT (Kirsch et al., 2005) appear to involve the amygdala. Bilateral lesions of the human amygdala eliminate emotion-memory interactions (Cahill et al., 1995; Strange et al., 2003; Adolphs et al., 2005a; Hurlemann et al., 2007) and impair emotion recognition (Adolphs et al., 1994, 2005b; Anderson and Phelps, 2001) and social judgment abilities (Adolphs et al., 1998). The amygdala is also implicated in empathic responses (Dziobek et al., 2008). We therefore investigated whether the amygdala is involved in OT-sensitive aspects of the RALT and MET by comparing performance of two monozygotic twin patients with congenital Urbach-Wiethe disease and selective bilateral calcification damage to the amygdala against matched controls.

\section{Materials and Methods}

Subjects and protocols

Over the course of 36 months, we conducted a series of three related behavioral pharmacological and neuropsychological experiments involving 76 participants and two behavioral tasks (see Figs. $2 A, 3 A$ ). Written informed consent according to the latest revision of the 1964 Declaration of Helsinki was obtained from all participants before entry to the experiments, which were approved by the institutional research ethics board of the University of Bonn and exempted from clinical trial status. All healthy volunteers were right handed and determined to be free of current or past physical (including daltonism) or psychiatric illness by medical history and diagnoses according to the Structured Clinical Interview for DSM-IV-TR (Diagnostic and Statistical Manual of Mental Disorders). They had no history of drug or alcohol abuse and did not report a prior use of psychoactive medication including OT.

The rationale of experiment 1 was to assess the effects of OT on both the RALT and the MET in a between-subject, double-blind, randomized, placebo-controlled investigation involving 48 healthy male volunteers $(n=24$ placebo, mean \pm SD age $25.2 \pm 2.5$ years; $n=24$ OT, age $26.7 \pm$ 2.2 years) (for neuropsychological screening, see supplemental Table S1, available at www.jneurosci.org as supplemental material). Consistent with previous studies (Kosfeld et al., 2005; Baumgartner et al., 2008; Guastella et al., 2008a,b; Rimmele et al., 2009), subjects were administered intranasal OT (24 IU; Syntocinon-Spray, Novartis; 3 puffs per nostril, each with 4 IU OT) or placebo (identical sprays without the active ingredient) $45 \mathrm{~min}$ before testing. Both tasks were tested on the same day with half the subjects receiving the RALT first and half the MET first. In this way, each subject either received placebo or OT twice during the experiment with a period of $2 \mathrm{~h}$ between treatments. The duration of the two tasks was $\sim 30 \mathrm{~min}$ for the RALT and $45 \mathrm{~min}$ for the MET. The decision to give treatments twice rather than once was prompted both by a previous study that only confirmed elevated CSF levels of neuropeptides up to $80 \mathrm{~min}$ after intranasal infusion (Born et al., 2002) and by an animal study that showed CSF OT levels fall considerably by $2 \mathrm{~h}$ after large increases seen at birth (Kendrick et al., 1991). The total OT dose of $48 \mathrm{IU}$ given was similar to the maximum single effective dose given in previous studies (Zak et al., 2007). Blood pressure and pulse rate were monitored in all subjects and there were no significant effects of OT.

In experiment 2, we tested monozygotic twin women, patients 1 and 2, with selective and bilaterally symmetrical amygdala calcification damage due to lipoid proteinosis of Urbach-Wiethe on the RALT. Sixteen female control volunteers matched for age and IQ served as the comparison group [for already published sample demographics and neuropsychological screening, see Hurlemann et al. (2007, 2009)].

In experiment 3 , we tested the same two patients for their performance on the MET. Given a 30 month interval between experiments 2 and 3, and in view of the slowly progressive nature of intracranial mineralizations in Urbach-Wiethe (UW) disease (Appenzeller et al., 2006), we once again scanned the patients using cranial computer tomography (CCT) (Fig. 1) and reassessed them both clinically and neuropsychologically. Of the 20 new candidates recruited as potential healthy controls, an age(patients 1 and 2 current age 34.8 years; $n=10$ controls, mean \pm SD age $35.5 \pm 3.9$ years) and IQ-matched group of 10 was selected for statistical comparisons.

Neuropsychological assessment of Urbach-Wiethe patients 1 and 2 Clinical symptomatology. Lipoid proteinosis of UW is a rare autosomal recessive disorder typified by cutaneous, mucosal, and visceral deposits of periodic acid-Schiff-positive hyaline (glycoprotein) material that pathognomonically presents itself in early infancy through hoarse cries due to laryngeal infiltration and follows a slowly progressive, yet often benign course. UW has been mapped to a locus on chromosome 1q21, and pathogenic 
loss-of-function mutations have been identified within the extracellular matrix protein 1 gene (ECM1) (Hamada, 2002; Chan et al., 2007). Selective bilateral calcification damage to the amygdala occurs in $50-75 \%$ of UW cases and has been shown to cause impaired fear recognition from facial expressions, reduced fearfulness in social contexts, and a failure to acquire conditioned fear responses (Adolphs et al., 1994, 1998, 2005). UW is frequently associated with epilepsy (Appenzeller et al., 2006). Whereas patient 1 has never suffered epileptic seizures, patient 2 suffered an epileptic grand-mal seizure at age 12 , which led to diagnosis of UW and subsequent diagnosis of her twin sister. Due to pregnancy, she has stopped her anticonvulsive treatment with a $900 \mathrm{mg}$ daily dose of valproate in 2006. Since the last testing in which the patients performed normally in the neuropsychological test battery (Hurlemann et al., 2007, 2009), both patients now report preepileptic auras that occur once or twice a month. This increase in clinical symptomatology is consistent with the results of latest CCT, showing that bilateral calcification lesions now symmetrically span the whole amygdala region rather than primarily affecting the basolateral subregion (BLA) alone (Hurlemann et al., 2007) (Fig. 1). This finding is consistent with the observation that intracranial mineralizations in UW are slowly progressive in nature (Appenzeller et al., 2006). Given these changes in clinical symptomatology, it was necessary to test both patients again with a comprehensive neuropsychological test battery.

Test battery. We used an extensive neuropsychological battery to test the patients for memory, attention, IQ, and visuospatial construction skills, as well as screen them for psychopathologies. The RAVLT (Rey Auditory Verbal Learning Test) (Rey, 1941, 1964; Helmstaedter et al., 1981) was used to assess immediate verbal memory span, new learning, susceptibility to interference, and delayed recall. The CFT (Rey-Osterrieth Complex Figure Test) was included to test incidental visual memory as well as the visuospatial constructional ability (Rey, 1941; Osterrieth, 1944). To examine figural learning and memory, we used the DCS ("Diagnosticum für Cerebralschädigung") developed for brain-damaged patients (Weidlich and Lamberti, 2001). Furthermore, speed of attention and mental flexibility were assessed with the TMT (Trail Making Test) (Reitan, 1955), and short-term concentration ability was tested with the nonverbal $\mathrm{d} 2$ test ("Aufmerksamkeits- und Belastungstest d2") (Brickenkamp, 1995). Color-word interference was tested with the Stroop test (Stroop, 1935; Baeumler, 1985). To assess perseveration and abstract reasoning, we included the WCST-64, a normed modification of the Wisconsin Card Sorting Test that uses one deck of cards (Kongs et al., 2000). To assess speed and flexibility of verbal thought processes, we included the RWT ("Regensburger Wortflüssigkeitstest”), a verbal fluency test (Aschenbrenner et al., 2000). The HAWIE-R ("Hamburg-Wechsler Intelligenztest für Erwachsene in revidierter Fassung”), a German-language adaptation of the WAIS-R (Wechsler Intelligence Test for Adults-Revised) (Wechsler, 1981; Tewes, 1991) provided a measure of verbal, performance, and fullscale IQ; the Mehrfachwahl-Wortschatztest provided a verbal IQ (Lehrl, 1978). Nonverbal reasoning IQ was assessed with the LPS-4 ("Leistungsprüfsystem" subtest 4) (Horn, 1983). The BDI-II (Beck Depression Inventory-Second Edition) (Beck et al., 1996), the HDRS-21 (Hamilton Depression Rating Scale) (Hamilton, 1960), and the HARS (Hamilton Anxiety Rating Scale) (Hamilton, 1959) were used for depression and anxiety screening. For further assessment of psychological and psychopathological symptom load, we included the SCL-90-R (Symptom Checklist-90-Revised) instrument (Derogatis, 1993; Franke,
1995). Perception and understanding of nonverbal communicative signals of emotion (facial expressions/tone of voice) were assessed with the TAB ("Tübinger Affekt Batterie"), a Germanlanguage version of the FAB (Florida Affect Battery) (Bowers et al., 1991; Breitenstein et al., 1996).

Results and interpretation. Patient 1 showed normal immediate memory, efficiency of learning, verbal interference control, and delayed recall in the RAVLT. Her attention skills (TMT Part A, d2) and cognitive flexibility (TMT Part B) were also normal, as well as her cognitive interference control (Stroop), semantic fluency (RWT), and visuospatial construction ability (CFT). Patient 1's cognitive flexibility (WCST-64) was normal to mildly impaired, and her figural (CFT delayed recall) and recognition memory (RAVLT) were mildly impaired. Patient 1 ranged from mildly to moderately impaired in learning capacity (DCS), and moderately impaired in phonetic fluency (RWT) (supplemental Table S2, available at www.jneurosci.org as supplemental material). Patient 1's twin sister, patient 2, showed normal immediate memory, efficiency of learning, verbal interference control, delayed recall, and recognition in the RAVLT. Her figural memory (CFT delayed recall), learning capacity (DCS), attention skills (TMT Part A, d2), cognitive flexibility (TMT Part B), cognitive interference control (Stroop), visuospatial construction (CFT), and cognitive flexibility (WCST-64) were also normal. In addition, patient 2 displayed normal verbal and performance IQ (HAWIE-R). Patient 2's semantic fluency (RWT) was between normal to mildly impaired. Her phonetic fluency (RWT) was moderately impaired (supplemental Table S2, available at www. jneurosci.org as supplemental material). Patients 1 and 2 both performed normal on the majority of IQ tests (HAWIE-R, MWT-B, LPS-4) (supplemental Table S3, available at www. jneurosci.org as supplemental material). They were euthymic (HDRS-21, HARS, BDI-II) and did not suffer from psychopathological symptoms (SCL-90-R) (supplemental Table S4, available at www.jneurosci.org as supplemental material). However, both patients were considerably compromised in facial emotion discrimination (TAB), when compared to the 10 females matched for age (mean \pm SD age $35.5 \pm 3.86$ ) and IQ (mean LPS-4 nonverbal reasoning IQ 107.5; mean MWT-B verbal IQ 104.5), who also served as control subjects for the MET (supplemental Table S5, available at www.jneurosci.org as supplemental material). In conclusion, both patients exhibit common limited neuropsychological impairments, which, however, do not significantly differ from their previous results (Strange et al., 2003; Hurlemann et al., 2007, 2009) and do not reflect their differential performance on the MET. Overall, patients 1 and 2 show a progress of their amygdala degeneration, however, without changes in their neuropsychological profile.

\section{Behavioral paradigms}

$R A L T$. We devised a behavioral paradigm that required subjects to make push-button responses to judge the category membership " $A$ " or "B" of three-digit numerical items presented repeatedly on a computer screen, with visual feedback immediately following each item-category judgment. Thus, knowledge of the correct or incorrect outcome of previous category judgments for a particular item served to enhance performance over subsequent trials. To avoid simple visuomotor learning (Wise and Murray, 1999), the response buttons for " $A$ " and " $B$ " changed depending on the random lateralization of " $\mathrm{A}$ " and "B" on the screen. In total, subjects completed four blocks, with eight trials (including four items of each category) presented over six cycles during each of these blocks. Within each cycle, trials were presented in random order. Hence, the number of trials per block was 48 , leading to 192 trials over the entire 
A

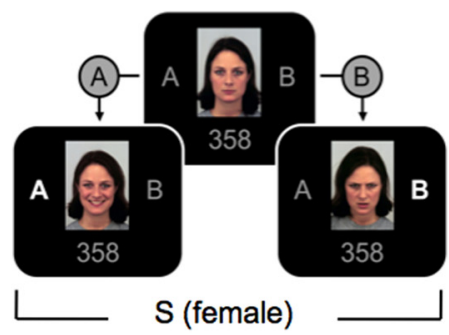

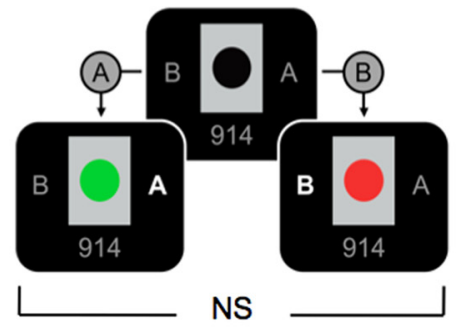

NS
B (i)

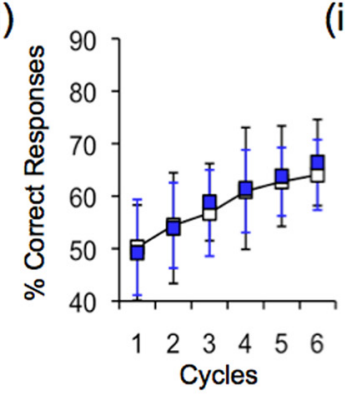

C

(i)

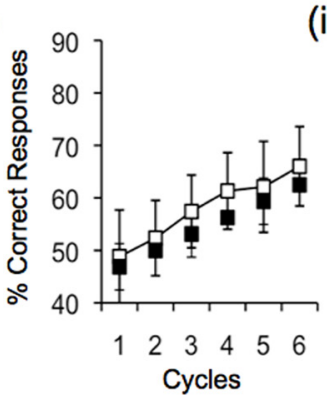

(ii)

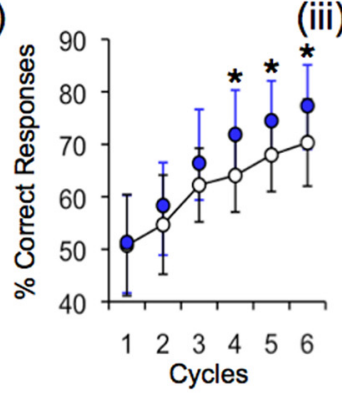

(ii)

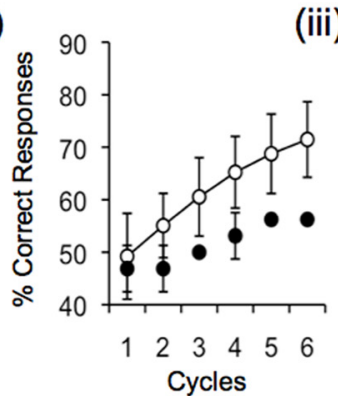

(iii)

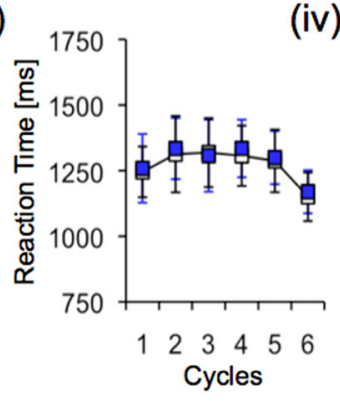

(iv)

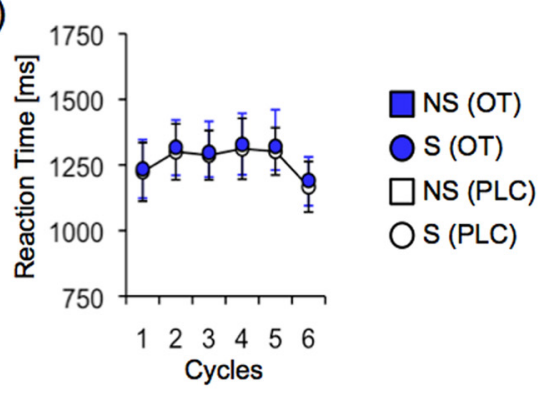

(iii)

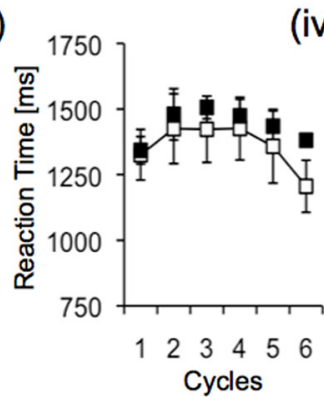

(iv)

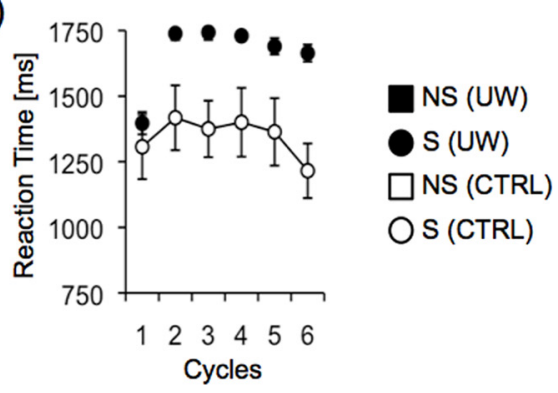

Figure 2. RALT. $\boldsymbol{A}$, Letters " $\mathrm{A}$ " and " $\mathrm{B}$ " flanked either a female or a male facial display in social trials and a black circle in nonsocial trials. Subjects judged whether three-digit numerical items presented repeatedly on a computer screen belonged to either category " $A$ " or " $B$ "; visual feedback immediately followed each judgment. Neutral faces changed to happy for correct responses or to angry for incorrect responses in the social condition (S). Black circles changed to green for correct responses or to red for incorrect responses in the nonsocial condition (NS). Bi, Percentage correct responses for the nonsocial condition did not differ between male subjects treated with $0 \mathrm{~T}$ ( $n=24$ ) or placebo (PLC, $n=24$ ). Bii, 0 xytocin increased overall performance up to $27.3 \pm 7.8 \%$ above chance level in male subjects, compared to $20.3 \pm 8.3 \%$ above chance for placebo controls in the social condition $\left({ }^{*} p<0.05\right)$. Biii, Reaction times for the nonsocial condition did not differ between male subjects treated with oxytocin or placebo. Biv, Reaction times for the social condition did not differ between male subjects treated with oxytocin or placebo. Ci, Percentage correct responses did not differ between two UW patients and controls (CTRL, $n=16$ ) for the nonsocial condition. Cii, Percentage correct responses decreased in UW patients compared to controls for the social condition (see Results section for Z scores). Ciii, Reaction times did not differ between UW patients and controls for the nonsocial condition. Civ, Reaction times were increased for UW patients compared with controls for the social condition. Significance threshold ${ }^{*} p<0.05$ indicates differences between groups. Error bars indicate SEM.

paradigm. In half of the blocks, subjects were exposed to social trials in which the letters " $A$ " and "B" flanked either a female or a male facial display; in the remaining blocks nonsocial trials were presented, with " $\mathrm{A}$ " and "B" flanking a black circle. The order of blocks alternated, with the first being social in half of the subjects. Social and nonsocial trials were identical in all aspects of spatial configuration and timing, with a trial duration of $3 \mathrm{~s}$ (stimulus-response duration, $2 \mathrm{~s}$; feedback duration, $1 \mathrm{~s}$ ) and a jittered intertrial interval of $5.5(3-8)$ s. Subjects were informed that there was no underlying rule defining which item belonged to category "A" or "B," and that category membership of each item was based on an arbitrary and randomized algorithm before the start of the task. Once assigned, category membership remained constant over six presentations (cycles). At the first cycle, subjects had no knowledge of the category membership and thus responded by guessing. Depending on whether a particular item-category judgment was right or wrong, facial displays changed from neutral to happy for correct responses or from neutral to angry for incorrect responses in the social trials. In contrast, black circles changed to green for correct responses or to red for incorrect responses in the nonsocial trials (Fig. 2A). This paradigm thus incorporated two conditions in which social-facilitative effects have been observed: first, the mere presence of others (Allport, 1920) — a female or male observer onscreen-and second, reinforcing (encouraging vs discouraging) feedback from others (Gates and Rissland, 1923; Zajonc, 1965)_changes in the observer's facial expression toward either rewarding (smiling) or punishing (angry) guiding judgments in subsequent trials. This design is consistent with current concepts that positive facial emotion increases the probability of a particular behavior, whereas negative facial emotion decreases it on future occasions (Blair, 2003). Nonsocial trials lacked this specific social-motivational input and thus served as a control.

$M E T$. An extended version of the MET was used to that previously described (Dziobek et al., 2008). The task allowed differentiation of cognitive and emotional facets of empathy. A total of 40 pictures showing people in emotionally charged situations served as ecologically valid stimuli (Fig. $3 A$ ), i.e., they depicted everyday life scenes conveying information on emotional mental states via facial expressions, body language, and context. To assess cognitive empathy $(C)$, participants were required to infer the mental state of the individual in each scene, and indicated the correct one from a list of four alternatives by push-button responses. For emotional empathy, participants were required to rate on a 1-9 intensity scale of how much they were feeling for the individual in each scene (ED) or how much they were aroused by each scene (EI). Each category tested for a positive and negative valence, thus resulting in six distinct data subsets $(\mathrm{C}+, \mathrm{C}-, \mathrm{ED}+, \mathrm{ED}-, \mathrm{EI}+$, and $\mathrm{EI}-)$ with a total of 120 questions (20 for each of the six categories). MET completion requires $\sim 45 \mathrm{~min}$. 


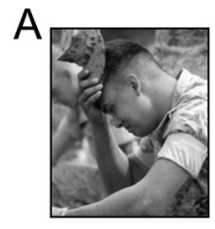

B
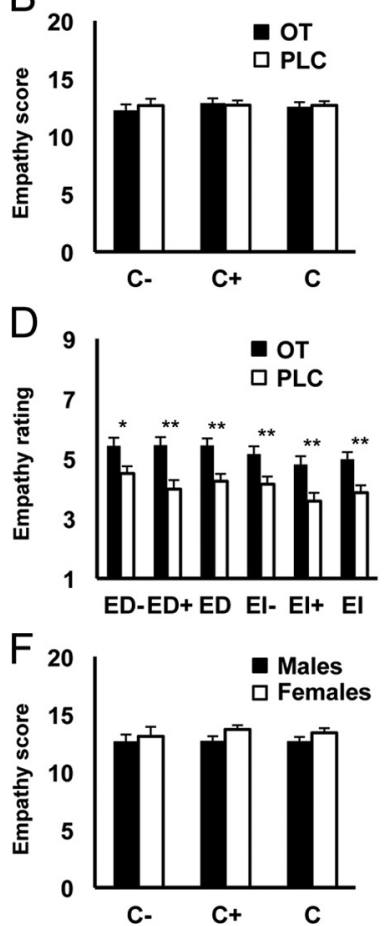
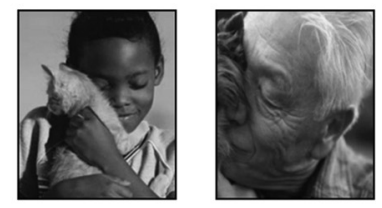

C

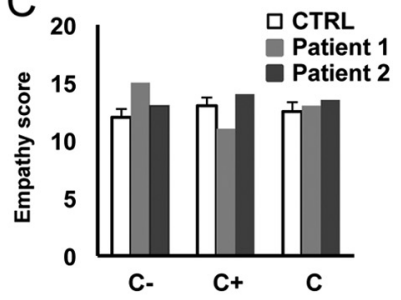

E
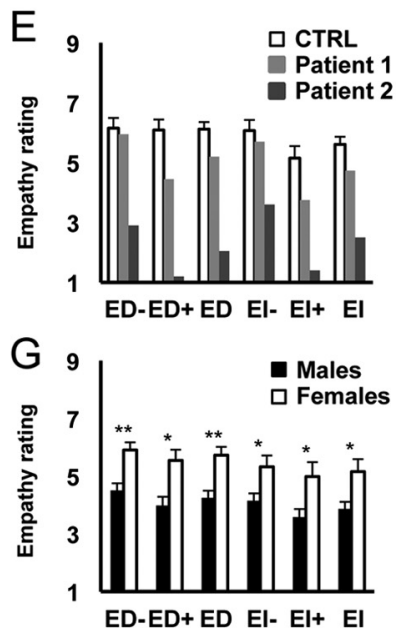

Figure 3. MET. A, Given are four example pictures taken from the MET to test for negative (left two scenes) and positive (right two scenes) empathy valence. To assess cognitive empathy $(C)$, participants were required to infer the mental state of the individual in each scene, and indicated the correct one from a list of four alternatives by push-button responses. For emotional empathy, participants were required to rate on a 1-9 intensity scale of how much they were feeling for the individual in each scene (ED) or how much they were aroused by each scene (El). $\boldsymbol{B}$, Cognitive empathy scores ( $C-=$ negative valence; $C+=$ positive valence; $C=$ positive and negative combined) did not differ between male subjects treated with $0 \mathrm{~T}(n=24)$ and placebo controls (PLC; $n=24)$. C, Cognitive empathy scores did not differ between controls (CTRL, $n=10$ ) and both UW patients, patients 1 and 2. D, Emotional empathy ratings were increased across all categories [direct emotional empathy, negative valence (ED-), positive valence $(E D+)$, and overall (ED); indirect emotional empathy, negative valence $(E I-)$, positive valence $(E I+)$, and overall $(E I)]$ in male subjects treated with $0 \mathrm{~T}(n=24)$ compared to placebo (PLC, $n=24)$. $\boldsymbol{E}$, Emotional empathy ratings were decreased across all emotional empathy categories (as in $\boldsymbol{D}$ ) for both UW twins, but particularly for patient 2, compared with controls (CTRL, $n=10)$ ( $Z$ scores are given in the results section). $F$, Cognitive empathy scores did not differ between women $(n=10)$ and men $(n=24)$. $\mathbf{G}$, Emotional empathy ratings were increased across all categories (as in $\boldsymbol{D}$ ) for women compared to men. Significant betweengroup differences: ${ }^{* *} p<0.01,{ }^{*} p<0.05$. Error bars indicate SEM.

\section{Results}

\section{RALT}

Oxytocinergic effects on RALT performance

For the experiment comparing intranasal OT- and placebotreated male subjects, a condition (nonsocial vs social reinforcement $\times$ cycles repeated-measures ANOVA restricted to the placebo group yielded main effects of condition $\left(F_{(1,23)}=5.641\right.$; $p=0.026)$ and cycles $\left(F_{(5,115)}=40.352 ; p<0.001\right)$, showing that learning with the social reinforcer $(20.3 \pm 8.3 \%$ above chance level after six cycles) was more effective than with the nonsocial reinforcer ( $14.0 \pm 6.7 \%$ above chance level after six cycles). A condition $\times$ cycles repeated-measures ANOVA with treatment group (placebo vs OT) as between-subjects factor yielded main effects of treatment $\left(F_{(1,46)}=8.639 ; p=0.005\right)$, condition $\left(F_{(1,46)}=\right.$ $28.943 ; p<0.001)$, and cycles $\left(F_{(5,230)}=94.506 ; p<0.001\right)$ as well as condition $\times$ treatment $\left(F_{(1,46)}=4.123 ; p=0.048\right)$ and condition $\times$ cycles $\left(F_{(5,230)}=3.524 ; p=0.004\right)$ interaction effects. An additional ANOVA with task order (MET, RALT) as covariate showed neither main nor interaction effects of task order, but still main effects of treatment $\left(F_{(1,45)}=8.66 ; p=0.005\right)$ and cycles $\left(F_{(5,225)}=8.044 ; p<0.001\right)$. In addition, there was a trend-to-significant condition $\times$ treatment interaction effect $\left(F_{(1,45)}=4.035 ; p=0.051\right)$.

Post hoc $t$ tests (using a Bonferroni correction for multiple comparisons) revealed that, relative to placebo treatment, OT selectively enhanced socially reinforced learning on cycles 4 $\left(+7.8 \% ; t_{(46)}=3.498 ; p=0.001 ; d=1.03\right), 5\left(+6.5 \% ; t_{(46)}=\right.$ $3.097 ; p=0.001 ; d=0.091)$, and $6\left(+7.0 \% ; t_{(46)}=3.029 ; p=\right.$ $0.004 ; d=0.089)$, resulting in an overall performance gain of $27.3 \pm 7.8 \%$ above chance level after six cycles. In addition, we observed a decrease in reaction times across cycles $\left(F_{(5,230)}=\right.$ 62.573; $p<0.001$ ) (Fig. 2 B). The number of missed responses did not differ between the placebo $(6.7 \pm 3.2 \%)$ and the OT $(6.4 \pm$ $3.1 \%)$ treatment groups $(p>0.05)$.

\section{Importance of the amygdala for RALT performance}

To assess dependence of social reinforcement learning on amygdala function, comparisons were made between the UW twins and 16 controls matched for sex, age, IQ, and education (Hurlemann et al., 2007, 2009). Because the patients' results overlapped ( $S D=0$ ), they could be collapsed into a mean score and compared to the mean controls. An initial condition $\times$ cycles repeated-measures ANOVA restricted to the control group yielded main effects of condition $\left(F_{(1,15)}=4.706 ; p=0.047\right)$ and cycles $\left(F_{(5,75)}=48.258\right.$; $p<0.001)$, confirming that learning with the social reinforcer (21.5 $\pm 7.2 \%$ above chance level after six cycles) was better than with the nonsocial reinforcer $(16.0 \pm 7.6 \%$ above chance level after six cycles). In the nonsocial reinforcement condition, UW patients displayed only minor performance deficits (12.5\% above chance level after six cycles; $Z=-0.47$ ), but were considerably impaired in the social reinforcement condition (only $6.25 \%$ above chance level after six cycles; $Z=-2.11$ ). Response times for UW patients were also much slower in the social reinforcement condition, with $Z$ scores ranging between -2.60 (second cycle) and -4.31 (sixth cycle) (Fig. $2 C$ ), and they missed more responses than did controls in the social reinforcement condition $(+8.52 \% ; Z=-6.22)$. These results strongly suggest that the bilateral amygdala lesion in the UW patients prevented them from effective feedback-guided learning with social but not nonsocial reinforcement.

\section{MET}

Oxytocinergic effects on MET ratings

Separate two-way ANOVAs were used to analyze the cognitive and emotional empathy data, with treatment and task order as factors. There was no OT treatment effect on overall cognitive empathy scores $\left(F_{(1,46)}=0.059, p=0.809\right)$, or on either negative $\left(\mathrm{C}-, F_{(1,46)}=0.286 ; p=0.595\right)$ or positive $\left(\mathrm{C}+, F_{(1,46)}=0.088\right.$; $p=0.768$ ) valence components (Fig. $3 B$ ). There were also no significant task order or order $\times$ treatment effects. There was an overall OT treatment effect for combined ED intensity ratings $\left(F_{(1,46)}=14.46 ; p<0.0001\right)$ and for both negative $\left(\mathrm{ED}-, F_{(1,46)}=\right.$ 7.132; $p=0.011)$ and positive $\left(\mathrm{ED}+, F_{(1,46)}=14.672 ; p<\right.$ 0.0001 ) valence stimuli (Fig. $3 D$ ). While significant order effects were absent, there was an overall treatment $\times$ order effect $(E D$, 
$\left.F_{(1,46)}=9.734 ; p=0.003\right)$ and for both the negative $\left(\mathrm{ED}-, F_{(1,46)}=\right.$ $8.065 ; p=0.007)$ and positive $\left(\mathrm{ED}+, F_{(1,46)}=6.539 ; p=0.014\right)$ valences. This indicated that subjects receiving OT for a second time before the MET showed a reduced effect. For combined EI intensity ratings there was also an overall treatment effect (EI, $\left.F_{(1,46)}=11.01 ; p=0.002\right)$ and for both negative $\left(F_{(1,46)}=7.945 ; p=\right.$ $0.007)$ and positive $\left(F_{(1,46)}=9.93 ; p=0.003\right)$ valence stimuli. There were no significant task order effects but there was a trend-to-significant treatment $\times$ order effect $\left(\mathrm{EI}, F_{(1,46)}=\right.$ $3.775 ; p=0.058 ; \mathrm{EI}-, F_{(1,46)}=3.868 ; p=0.056$; EI,$+ F_{(1,46)}=$ $2.415 ; p=0.127)$.

\section{Importance of the amygdala for MET ratings}

For the two UW patients, overall cognitive empathy scores were similar to controls (C, patient $1, Z=0.39$; patient $2, Z=0.79$ ). Their positive valence scores did not differ from controls $(\mathrm{C}+$, patient $1, Z=-0.88$; patient $2, Z=0.44$ ), although for negative valence, patient 1 had a moderately improved score $(\mathrm{C}-$, patient $1, Z=1.33$; patient $2, Z=0.44$ ) (Fig. $3 C$ ). On the other hand, patient 2 was strongly impaired on both direct (ED, $Z=-5.39$; $\mathrm{ED}-, Z=-2.98 ; \mathrm{ED}+, Z=-4.45)$ and indirect $(\mathrm{EI}, Z=-3.86$; $\mathrm{EI}-, Z=-2.18$; $\mathrm{EI}+, Z=-2.93$ ) empathy intensity ratings regardless of valence, whereas patient 1 was only moderately impaired on overall and positive valence ratings but not on negative valence ones (ED, $Z=-1.20$; $\mathrm{ED}-, Z=-0.18$; $\mathrm{ED}+, Z=$ -1.49; EI, $Z=-1.09$; EI,$- Z=-0.33$; EI,$+ Z=-1.10$ ) (Fig. $3 E)$. Although patient 1's results also showed lower empathy ratings than controls, her $Z$ scores were lower than patient 2 's.

\section{Gender differences in $M E T$ ratings}

A comparison was made between the 10 women with higher IQs in the original control group for experiment 3 and the 24 placebo-treated men with similar IQs in the OT experiment to investigate potential gender differences in the MET. Cognitive empathy scores did not significantly differ between the sexes ( $t$ test with Bonferroni correction-C, $t_{(32)}=-1.36 ; p=0.21 ; \mathrm{C}-$, $t_{(32)}=0.40 ; p=0.69 ; \mathrm{C}+, t_{(32)}=1.47 ; p=0.15$ ) (Fig. $3 F$ ), but women had significantly higher emotional empathy intensity ratings for both $\mathrm{ED}\left(\mathrm{ED}, t_{(32)}=3.41 ; p=0.003 ; d=1.32 ; \mathrm{ED}-, t_{(32)}=\right.$ $3.30 ; p=0.004 ; d=1.28 ; \mathrm{ED}+, t_{(32)}=2.90 ; p=0.013 ; d=1.13$ ) and $\mathrm{EI}\left(\mathrm{EI}, t_{(32)}=2.65 ; p=0.025 ; d=1.03 ; \mathrm{EI}-, t_{(32)}=2.45 ; p=\right.$ $0.04 ; d=0.95 ; \mathrm{EI}+, t_{(32)}=2.51 ; p=0.035 ; d=0.97$ ) (Fig. $3 G$ ). The emotional empathy ratings for OT-treated men did not significantly differ from those of control women $(p>0.05$ in all cases). On average the female subjects were 8 years older than the males (33.7 vs 25.2 years), but there were no significant interactions between gender and age on any of the MET parameters $\left(\mathrm{ANCOVA}-\mathrm{C}, F_{(1,30)}=1.13, p=0.30 ; \mathrm{ED}, F_{(1,30)}=2.21, p=\right.$ 0.15 ; EI, $\left.F_{(1,30)}=0.94, p=0.339\right)$. There were also no overall significant correlations between age and performance on total cognitive or emotional empathy $t$ scores in the MET in either group, again suggesting that this age difference is unlikely to have selectively increased emotional empathy intensity ratings (correlations between scores and age-females, $\mathrm{C}, r=-0.07$; ED, $r=-0.13$; EI, $r=-0.03$; males, C, $r=0.29$; ED, $r=0.36$; EI, $r=0.31 ; p>0.05$ in all cases). There were also no positive correlations between age and performance on negative or positive components on the MET $(p>0.05$ in all cases), with the exception of $\mathrm{C}+$ in males $(r=0.59, p<0.01)$. A further comparison between the 10 women with higher IQs used in this analysis (Fig. $3 G$ ) and the 10 with lower IQs used in experiment 3 (Figs. 3C,E) revealed no significant differences between the two groups for any aspect of cognitive or emotional empathy scores
( $p>0.05$ in all cases). Thus it would appear that IQ is unlikely to be of importance in relation to performance or gender differences in the MET.

\section{Discussion}

Our results provide the first evidence that OT selectively enhances socially reinforced learning. Not only does this confirm that social reinforcement of learning is important (Allport, 1920; Gates and Rissland, 1923; Dashiell, 1930; Zajonc, 1965), but also extends the cognitive enhancement effects of OT beyond social identity or emotion recognition shown in humans (Domes et al., 2007a,b; Rimmele et al., 2009; Guastella et al., 2008a,b) and other species (Dantzer et al., 1987; Kendrick et al., 1987; Lim and Young, 2006) to other forms of learning, provided social reinforcement is used. Clearly it will be relevant to investigate this using other examples of learning tasks. Our findings, together with those of facilitated social recognition memory, contrast with studies reporting amnestic effects of OT (Ferrier et al., 1980; Fehm-Wolfsdorf et al., 1984; Bruins et al., 1992; Heinrichs et al., 2004), which often involve highly aversive and stressful experimental contexts (de Wied, 1965; Wu and Yu, 2004; Petrovic et al., 2008). Indeed, it seems possible that OT may selectively enhance learning where there is some prosocial component but aid forgetting where the overall learning context is experienced as highly aversive and stressful.

Both UW twins displayed no social reinforcement facilitation of learning in our study, suggesting that a functional amygdala may indeed be important in mediating these effects. The neuropsychological profiles show that patients 1 and 2 exhibit common limited neuropsychological impairments, which do not significantly differ from previous examinations (Strange et al., 2003; Hurlemann et al., 2007, 2009). Although both UW twins were severely impaired in discriminating between facial emotions, patients 1 and 2 were not compromised in identifying facial emotions per se and did not report significant impairment in distinguishing the positive and negative feedback faces in the RALT. It seems likely, therefore, that the observed decreases in performance were due to patients having to develop a more laborious cognitive strategy to recognize the different facial emotions used for feedback. This compensational strategy could have used other brain regions such as the prefrontal cortex. The amygdala damage per se may, however, have interfered with the ability to ascribe motivational value to the emotional feedback faces, thereby preventing social reinforcement effects. The UW twins furthermore showed increased reaction times compared to the OT-treated subjects. Here again, it seems likely that the amygdala degeneration resulted in a necessarily more laborious cognitive process than the healthy subjects given OT used to arrive at the same decision.

Other studies have also implicated the amygdala as an important site of action for OT in facilitating recognition of, or memory for, faces and facial emotions in humans (Guastella et al., 2008b; Savaskan et al., 2008; Rimmele et al., 2009). The amygdala is also known to play a key role in OT facilitation of olfactory recognition of individuals in rodents (Ferguson et al., 2001). These findings, together with those reported here, contrast with one report that intranasal OT reduces amygdala responses to both emotional and nonemotional faces (Domes et al., 2007b). However, another study has reported reduced amygdala activation only during exposure to fear-evoking faces or scenes (Kirsch et al., 2005), and therefore OT may facilitate amygdala activity under positive, prosocial/empathy-provoking conditions and inhibit it under more aversive and stressful conditions where stimuli are 
avoided (see also Petrovic et al., 2008). While it might be argued that the angry faces used in our study to signal errors to the subjects are also aversive, they were shown for a minority of the time during the trials compared with the neutral and smiling versions of the same face. Interestingly, a recent study has reported increased amygdala activation in response to fearful faces in women, raising the possibility that there might be a gender difference in the amnestic effects of OT during aversive social contexts (Domes et al., 2010). Indeed, in rats it has been reported that an OTR antagonist can both excite and inhibit the activity of different cell populations in the central amygdala (Huber et al., 2005).

Our results also provide a first demonstration for OT-induced facilitation of self-reported emotional empathy intensity ratings in the MET in men for both positive and negative valence stimuli, although the reduced effects following repeated OT treatment suggest that higher doses of the neuropeptide might not be as effective, perhaps due to OTR desensitization. Our findings contrast with a previous functional imaging study in men, which did not find such effects using empathic responses to pain experienced by female partners (Singer et al., 2008). The authors concluded that their failure to find OT effects might be because empathic responses to pain are not amygdala dependent. However, the MET used in our current study tests for empathic responses to individuals expressing specific positive or negative emotional states rather than physical pain and does appear to have some amygdala involvement since the UW patients were impaired. The observation that the UW twins were not compromised in cognitive empathy, but showed a moderate to severe impairment in emotional empathy, further supports the arguments made above in interpreting their RALT performance. They seem quite capable of identifying facial emotions by cognitive strategies, but in the absence of a functional amygdala, they are impaired in processing and responding to the emotional consequences of this recognition. We suggest that the seemingly contrasting findings are not necessarily polar opposites: While OT failed to influence empathy for a loved one experiencing pain (Singer et al., 2008), thus failing to influence behavior to an extremely aversive and stressful experimental stimulus, we used pictures of faces and social scenes lacking this intensity of aversive and stressful stimulation, and this might have resulted in OT being more effective. Alternatively, as Singer et al. (2008) suggest, it may simply be that empathy for pain does not involve the amygdala.

One limitation to our study in this context is the difference in emotional empathy ratings between the UW twins, who show variation in emotional response to different valences, as well as degree of emotional response compared to controls. Patient 1 was less impaired in this respect than patient 2 ; the fact that patients 1 and 2 are monozygotic twins suffering from a genetic disorder argues against a potential influence of dissociable etiologies. In addition, there is no radiological evidence for differences in lesion size. It thus appears most likely that the observed discrepancies are due to epigenetic factors differentially influencing emotional functions including empathy, either directly or indirectly via the plastic compensational adaptations likely associated with amygdala degeneration in early infancy (Hurlemann et al., 2007, 2009).

Our failure to find any effects of OT or amygdala dependence for cognitive empathy performance in the MET appears to contrast with a previous study using the "reading the mind in the eyes test" (RMET) in which intranasal OT selectively improved performance on difficult test items (Domes et al., 2007a). This latter task requires subjects to infer internal emotional state of another individual from subtle differences in the eyes and also activates the amygdala (Baron-Cohen et al., 1999). Eye gaze monitoring is amygdala dependent and reported to be impaired in UW patients (Adolphs et al., 1994, 2005). However, in the MET the eyes of the stimulus subjects are only part of the information displayed, with other facial features, body language, and even social context being present. Another study has shown that OT increases the amount of time looking at the eyes in faces (Guastella et al., 2008a), and this may have contributed indirectly to improved performance on the RMET (Domes et al., 2007a) and explain why OT failed to improve cognitive empathy scores on the MET, where eye gaze perception is not of such exclusive importance. Thus, while impaired performance on both the RMET and cognitive empathy component of the MET have been reported in Asperger's syndrome (Dziobek et al., 2008) (see also Rogers et al., 2007), the neural contributions and the influence of OT in each task may be different.

Another major limitation to our study is the inability to determine whether amygdala function is or is not directly modulated by OT. What our study shows for the first time, however, is that social reinforcement learning and emotional empathy are both amygdala dependent and modulated by OT, suggesting that the amygdala is an essential part of a functional neurocircuitry mediating socially reinforced learning and emotional empathy, and that this functional neurocircuitry is susceptible to the modulatory action of OT.

While the current study was not designed to establish a gender difference in either the RALT or MET, the enforced use of both male and female control groups afforded this opportunity. Although there was an 8 year age difference between the male and female control groups, they performed identically on the learning task and the cognitive empathy component of the MET. However, the emotional empathy intensity ratings given by the women were considerably higher than men, and only when the men received OT treatment did they become equivalent to untreated women. Although we cannot completely rule out a contribution of age to this gender effect, we found no evidence for age $\times$ gender interactions across gender groups or correlations within them for emotional empathy ratings. This gender difference in emotional empathy is also in agreement with other studies (Toussaint and Webb, 2005) and may reflect enhanced release of OT in women compared with men (Taylor et al., 2000).

A general conclusion from our results is that treatment with an OTR agonist may be a useful therapy in enhancing socially motivated learning and emotional empathy in men. The potential for its use in helping to treat social and emotional impairments in autism spectrum disorders (ASDs) has been suggested by many, and is supported by a number of different findings. However, it is cognitive rather than emotional empathy that is reportedly impaired in individuals with ASD (Baron-Cohen et al., 1999; Dziobek et al., 2008), and our results show that OT treatment has rather minor effects in improving cognitive empathy. Augmenting the effectiveness of socially reinforced learning and levels of emotional empathy could, however, benefit the treatment of a number of disorders in which social-emotional responsivity is aberrant, such as schizophrenia (Derntl et al., 2009) or psychopathy (Kirsch and Becker, 2007).

By measuring performance on both the RALT and the MET, we have first provided evidence that intranasal OT treatment significantly enhances learning that is socially reinforced. Second, we have shown that OT treatment facilitates intensity judgment ratings on both direct and indirect negative and positive valence 
components of emotional empathy but not cognitive empathy scores. In addition, OT treatment increased emotional empathy ratings in men up to the higher level normally seen in untreated women. UW patients with bilateral amygdala lesion were impaired in the same OT-sensitive task components but not on non-socially reinforced learning or cognitive empathy, suggesting that the amygdala is essential part of a functional neurocircuitry that is modulated by OT. Whether this modulatory influence of OT is direct or indirect, and perhaps amygdala mediated, remains to be investigated by future studies.

\section{References}

Adolphs R (2009) The social brain: neural basis of social knowledge. Annu Rev Psychol 60:693-716.

Adolphs R, Tranel D, Damasio H, Damasio A (1994) Impaired recognition of emotion in facial expressions following bilateral damage to the human amygdala. Nature 372:669-672.

Adolphs R, Tranel D, Damasio AR (1998) The human amygdala in social judgment. Nature 393:470-474.

Adolphs R, Tranel D, Buchanan TW (2005a) Amygdala damage impairs emotional memory for gist but not details of complex stimuli. Nat Neurosci 8:512-518.

Adolphs R, Gosselin F, Buchanan TW, Tranel D, Schyns P, Damasio AR (2005b) A mechanism of impaired fear recognition after amygdala damage. Nature 433:68-72.

Allport FH (1920) The influence of the group upon association and thought. J Exp Psychol 3:159-182.

Anderson AK, Phelps EA (2001) Lesions of the human amygdala impair enhanced perception of emotionally salient events. Nature 411:305-309.

Appenzeller S, Chaloult E, Velho P, de Souza EM, Araújo VZ, Cendes F, Li LM (2006) Amygdalae calcifications associated with disease duration in lipoid proteinosis. J Neuroimaging 16:154-156.

Aschenbrenner S, Tucha O, Lange KW (2000) Regensburger Wortflüssigkeitstest. Göttingen, Germany: Hogrefe.

Baeumler G (1985) FWIT: Farbe-Wort-Interferenztest [The Stroop-Test]. Göttingen, Germany: Hogrefe.

Baron-Cohen S, Ring HA, Wheelwright S, Bullmore ET, Brammer MJ, Simmons A, Williams SC (1999) Social intelligence in the normal and autistic brain: an fMRI study. Eur J Neurosci 11:1891-1898.

Baumgartner T, Heinrichs M, Vonlanthen A, Fischbacher U, Fehr E (2008) Oxytocin shapes the neural circuitry of trust and trust adaptation in humans. Neuron 58:639-650.

Beck AT, Steer RA, Brown GK (1996) Manual for the Beck Depression Inventory-II. San Antonio: Psychological Corporation.

Blair RJ (2003) Facial expressions, their communicatory functions, and neuro-cognitive substrates. Philos Trans $\mathrm{R}$ Soc Lond B Biol Sci 358:561-572.

Born J, Lange T, Kern W, McGregor GP, Bickel U, Fehm HL (2002) Sniffing neuropeptides: a transnasal approach to the human brain. Nat Neurosci 5:514-516.

Bowers D, Blonder LX, Heilman KM (1991) Florida Affect Battery. Gainesville, FL: University of Florida.

Breitenstein C, Daum I, Ackermann H, Lütgehetmann R, Müller E (1996) Erfassung der Emotionswahrnehmung bei zentralnervösen Läsionen und Erkrankungen: Psychometrische Gütekriterien der "Tübinger Affekt Batterie." Neurol Rehabil 2:93-101.

Brickenkamp R (1995) Aufmerksamkeitsbelastungstest 'd2', erweiterte und neu gestaltete Auflage. Diagnostica 41:291-296.

Bruins J, Hijman R, Van Ree JM (1992) Effect of a single dose of desglycinamide-[Arg8]vasopressin or oxytocin on cognitive processes in young healthy subjects. Peptides 13:461-468.

Cahill L, Babinsky R, Markowitsch HJ, McGaugh JL (1995) The amygdala and emotional memory. Nature 377:295-296.

Chan I, Liu L, Hamada T, Sethuraman G, McGrath JA (2007) The molecular basis of lipoid proteinosis: mutations in extracellular matrix protein 1 . Exp Dermatol 16:881-890.

Dantzer R, Bluthe RM, Koob GF, Le Moal M (1987) Modulation of social memory in male rats by neurohypophyseal peptides. Psychopharmacology (Berl) 91:363-368.

Dashiell JF (1930) An experimental analysis of some group effects. J Abnorm Soc Psych 25:190-199.
Derntl B, Finkelmeyer A, Toygar TK, Hülsmann A, Schneider F, Falkenberg DI, Habel U (2009) Generalized deficit in all core components of empathy in schizophrenia. Schizophr Res 108:197-206.

Derogatis LR (1993) The Brief Symptom Inventory: administration, scoring and procedures. Minneapolis: National Computer Systems.

de Wied D (1965) The influence of posterior and intermediate lobe of the pituitary and pituitary peptides on the maintenance of a conditioned avoidance response in rats. Int J Neuropharmacol 4:157-167.

Domes G, Heinrichs M, Michel A, Berger C, Herpertz SC (2007a) Oxytocin improves "mind-reading" in humans. Biol Psychiatry 61:731-733.

Domes G, Heinrichs M, Gläscher J, Büchel C, Braus DF, Herpertz SC (2007b) Oxytocin attenuates amygdala responses to emotional faces regardless of valence. Biol Psychiatry 62:1187-1190.

Domes G, Lischke A, Berger C, Grossmann A, Hauenstein K, Heinrichs M, Herpertz SC (2010) Effects of intranasal oxytocin on emotional face processing in women. Psychoneuroendocrinology 35:83-93.

Dziobek I, Rogers K, Fleck S, Bahnemann M, Heekeren HR, Wolf OT, Convit A (2008) "Dissociation of cognitive and emotional empathy in adults with Asperger syndrome using the Multifaceted Empathy Test (MET)." J Autism Dev Disord 38:464-473.

Fehm-Wolfsdorf G, Born J, Voigt KH, Fehm HL (1984) Human memory and neurohypophyseal hormones: opposite effects of vasopressin and oxytocin. Psychoneuroendocrinology 9:285-292.

Ferguson JN, Aldag JM, Insel TR, Young LJ (2001) Oxytocin in the medial amygdala is essential for social recognition in the mouse. J Neurosci 21:8278-8285.

Ferrier BM, Kennett DJ, Devlin MC (1980) Influence of oxytocin on human memory processes. Life Sci 27:2311-2317.

Franke GH (1995) SCL-90-R. Die Symptom Checkliste von Derogatis, Deutsche version. Göttingen, Germany: Beltz Test.

Gates GS, Rissland LQ (1923) The effect of encouragement and discouragement upon performance. J Educ Psychol 14:21-26.

Guastella AJ, Mitchell PB, Dadds MR (2008a) Oxytocin increases gaze to the eye region of human faces. Biol Psychiatry 63:3-5.

Guastella AJ, Mitchell PB, Mathews F (2008b) Oxytocin enhances the encoding of positive social memories in humans. Biol Psychiatry 64: $256-258$.

Hamada T (2002) Lipoid proteinosis. Clin Exp Dermatol 27:624-629.

Hamilton M (1959) The assessment of anxiety states by rating. Br J Med Psychol 32:50-55.

Hamilton M (1960) A rating scale for depression. J Neurol Neurosurg Psychiatry 23:56-62.

Hein G, Singer T (2008) I know how you feel but not always: the empathic brain and its modulation. Curr Opin Neurobiol 18:153-158.

Heinrichs M, Baumgartner T, Kirschbaum C, Ehlert U (2003) Social support and oxytocin interact to suppress cortisol and subjective responses to psychosocial stress. Biol Psychiatry 54:1389-1398.

Heinrichs M, Meinlschmidt G, Wippich W, Ehlert U, Hellhammer DH (2004) Selective amnesic effects of oxytocin on human memory. Physiol Behav 83:31-38.

Helmstaedter C, Lendt M, Lux S (1981) VLMT Verbaler Lern und Merkfähigkeitstest. Göttingen, Germany: Beltz Test.

Horn W (1983) L-P-S Leistungsprüfsystem. Göttingen, Germany: Hogrefe. Huber D, Veinante P, Stoop R (2005) Vasopressin and oxytocin excite distinct neuronal populations in the central amygdala. Science 308:245-248.

Hurlemann R, Wagner M, Hawellek B, Reich H, Pieperhoff P, Amunts K, Oros-Peusquens AM, Shah NJ, Maier W, Dolan RJ (2007) Amygdala control of emotion-induced forgetting and remembering: evidence from Urbach-Wiethe disease. Neuropsychologia 45:877-884.

Hurlemann R, Schlaepfer TE, Matusch A, Reich H, Shah NJ, Zilles K, Maier W, Bauer A (2009) Reduced 5-HT(2A) receptor signaling following selective bilateral amygdala damage. Soc Cogn Affect Neurosci 4:79-84.

Insel TR, Shapiro LE (1992) Oxytocin receptor distribution reflects social organization in monogamous and polygamous voles. Proc Natl Acad Sci U S A 89:5981-5985.

Kendrick KM, Keverne EB, Baldwin BA (1987) Intracerebroventricular oxytocin stimulates maternal behaviour in the sheep. Neuroendocrinology 46:56-61.

Kendrick KM, Keverne EB, Hinton MR, Goode JA (1991) Cerebrospinal fluid and plasma concentrations of oxytocin and vasopressin during parturition and vaginocervical stimulation in the sheep. Brain Res Bull 26:803-807. 
Kirsch LG, Becker JV (2007) Emotional deficits in psychopathy and sexual sadism: implications for violent and sadistic behavior. Clin Psychol Rev 27:904-922.

Kirsch P, Esslinger C, Chen Q, Mier D, Lis S, Siddhanti S, Gruppe H, Mattay VS, Gallhofer B, Meyer-Lindenberg A (2005) Oxytocin modulates neural circuitry for social cognition and fear in humans. J Neurosci 25:11489-11493.

Kongs SK, Thompson LL, Iverson GL, Heaton RK (2000) The Wisconsin Card Sorting Test (WCST-64): Computer Version 2 Research Edition. Odessa, FL: Psychological Assessment Resources.

Kosfeld M, Heinrichs M, Zak PJ, Fischbacher U, Fehr E (2005) Oxytocin increases trust in humans. Nature 435:673-676.

Lee HJ, Macbeth AH, Pagani JH, Young WS 3rd (2009) Oxytocin: the great facilitator of life. Prog Neurobiol 88:127-151.

Lehrl S (1978) Mehrfachwahl-Wortschatz-Intelligenztest MWT-B. Erlangen, Germany: Verlag Dr. med. Straube.

Lim MM, Young LJ (2006) Neuropeptidergic regulation of affiliative behavior and social bonding in animals. Horm Behav 50:506-517.

Osterrieth PA (1944) Le test de copie d'une figure complexe. Arch Psychol 30:206-356.

Petrovic P, Kalisch R, Singer T, Dolan RJ (2008) Oxytocin attenuates affective evaluations of conditioned faces and amygdala activity. J Neurosci 28:6607-6615.

Reitan RM (1955) The relation of the trail-making test to organic brain damage. J Consult Psychol 19:393-394.

Rey A (1941) L'examen psychologique dans les cas d'encephalopathie traumatique. Arch Psychol 28:286-340.

Rey A (1964) L'examen clinique en psychologie. Paris: Presses Universitaires de France.

Rimmele U, Hediger K, Heinrichs M, Klaver P (2009) Oxytocin makes a face in memory familiar. J Neurosci 29:38-42.

Rodrigues SM, Saslow LR, Garcia N, John OP, Keltner D (2009) Oxytocin receptor genetic variation relates to empathy and stress reactivity in humans. Proc Natl Acad Sci U S A 106:21437-21441.

Rogers K, Dziobek I, Hassenstab J, Wolf OT, Convit A (2007) Who cares? Revisiting empathy in Asperger syndrome. J Autism Dev Disord 37: $709-715$.

Savaskan E, Ehrhardt R, Schulz A, Walter M, Schächinger H (2008) Post- learning intranasal oxytocin modulates human memory for facial identity. Psychoneuroendocrinology 33:368-374.

Singer T, Snozzi R, Bird G, Petrovic P, Silani G, Heinrichs M, Dolan RJ (2008) Effects of oxytocin and prosocial behavior on brain responses to direct and vicariously experienced pain. Emotion 8:781-791.

Strange BA, Hurlemann R, Dolan RJ (2003) An emotion-induced retrograde amnesia in humans is amygdala- and beta-adrenergic-dependent. Proc Natl Acad Sci U S A 100:13626-13631.

Stroop JR (1935) Studies of interference in serial verbal reactions. J Exp Psychol 18:643-662.

Taylor SE, Klein LC, Lewis BP, Gruenewald TL, Gurung RA, Updegraff JA (2000) Biobehavioral responses to stress in females: tend-and-befriend, not fight and flight. Psychol Rev 107:411-429.

Tewes U (1991) Hamburg-Wechsler-Intelligenztest für Erwachsene, Revision. Bern, Switzerland: Hans Huber.

Thompson MR, Callaghan PD, Hunt GE, Cornish JL, McGregor IS (2007) A role for oxytocin and 5-HT(1A) receptors in the prosocial effects of 3,4methylenedioxymethamphetamine ("ecstasy"). Neuroscience 146:509-514.

Toussaint L, Webb JR (2005) Gender differences in the relationship between empathy and forgiveness. J Soc Psychol 145:673-685.

Veinante P, Freund-Mercier MJ (1997) Distribution of oxytocin- and vasopressin-binding sites in the rat extended amygdala: a histoautoradiographic study. J Comp Neurol 383:305-325.

Wechsler D (1981) Wechsler adult intelligence scale-revised. New York: The Psychological Corporation.

Weidlich S, Lamberti G (2001) DCS_Diagnosticum für Cerebralschädigung (DCS). Ein visueller Lern- und Ge-dächtnistest nach F. Hiller. Bern, Switzerland: Hans Huber.

Wise SP, Murray EA (1999) Role of the hippocampal system in conditional motor learning: mapping antecedents to action. Hippocampus 9:101-117.

Wu W, Yu L-C (2004) Roles of oxytocin in spatial learning and memory in the nucleus basalis of Meynert in rats. Reg Pep 120:119-125.

Yoshimura R, Kiyama H, Kimura T, Araki T, Maeno H, Tanizawa O, Tohyama M (1993) Localization of oxytocin messenger ribonucleic acid in the rat brain. Endocrinology 133:1239-1246.

Zajonc RB (1965) Social facilitation. Science 149:269-274.

Zak PJ, Stanton AA, Ahmadi S (2007) Oxytocin increases generosity in humans. PLoS ONE 2:e1128. 